\title{
Geometrische Betrachtung der Normalen, welche sich von einem beliebigen Punkte auf eine algebraische Fläche fällen lassen.
}

(Von Herrn F. August.)

Im $49^{\text {sten }}$ Bande dieses Journals giebt Steiner drei Methoden an, um die Anzahl der Normalen zu bestimmen, die sich im Allgemeinen von einem beliebigen Punkte aus auf eine Curve $n^{\text {ten }}$ Grades fällen lassen. Im Anschluss an die dritte Methode behandelt er dann das analoge Problem für die Normalen von einem beliebigen Punkte auf eine Oberfläche und erwähnt, dass dies auch nach der ersten Methode möglich sei, ohne indess Näheres hierüber anzugeben.

Diese erste Methode, die im Ganzen weniger Sätze der höheren Geometrie voraussetzt, ist hier auf die analoge Untersuchung bei der Fläche angewandt.

Die erste Steinersche Methode ist kurz folgende: Gegeben in einer Ebene eine Curve $C^{n}$ und ein beliebiger Punkt $\pi$. Denkt man sich die $C^{n}$ fest mit $\pi$ verbunden und unendlich wenig um $\pi$ gedreht, und nennt man die Curve in der neuen Lage $C_{1}^{n}$, so können sich $C_{1}^{n}$ und $C^{n}$ nur in solchen Punkten $\xi$ schneiden, für die $\pi \xi$ Normale von $C^{n}$ ist, und umgekehrt.

Es sind also die Schnittpunkte $\xi$ der Curven $C^{n}$ und $C_{1}^{n}$ die Fusspunkte aller Normalen von $\pi$ auf $C^{n}$. Ihre Anzahl ist $n^{2}$, und wenn $\frac{n(n+3)}{2}-1=\frac{n^{2}+3 n-2}{2}$ derselben bekannt sind, so sind die übrigen $\frac{n^{2}-3 n+2}{2}$ nach den bekannten Sätzen über den Durchschnitt algebraischer Curven zu finden.

Der Beweis kann sehr leicht durch die Betrachtung des Durchschnitts der Tangenten in zwei entsprechenden Punkten der Curven $C^{n}$ und $C_{1}^{n}$ geführt werden. Diesen Satz benutzen wir bei der Betrachtung der Flächen folgendermassen.

I.

Gegeben eine Fläche $n^{\text {ten }}$ Grades $\boldsymbol{f}^{n}$ und ein willkürlicher Punkt $\pi$. Wieviel Normalen lassen sich von $\pi$ auf $f^{n}$ fällen? Wir denken durch $\pi$ eine 
beliebige Gerade $A_{1}$ mit $f^{n}$ fest verbunden, die $f^{n}$ in den $n$ Punkten $\eta_{1}$ schneidet. Wir denken ferner $f^{n}$ um $A_{1}$ als Axe unendlich wenig gedreht in die Lage $f_{1}^{n}$, so schneiden sich $f^{n}$ und $f_{1}^{n}$ in einer Raumcurve $R_{1}^{n^{2}}=\left(f^{n} f_{1}^{n}\right)$, auf welcher auch die $n$ Punkte $\eta_{1}$ liegen. Legt man durch einen Punkt $\beta_{1}$ dieser Raumcurve eine Ebene $e_{1}$ normal gegen die Axe $A_{1}$, die dieselbe in $\alpha_{1}$ schneidet, so muss $\alpha_{1} \beta_{1}$ Normale in $\beta_{1}$ sein auf dem ebenen Schnitt $E_{l}^{n}=\left(f^{n} e_{1}\right)$ nach der obigen Betrachtung. Zieht man also in $\beta_{1}$ die Tangente $B_{1}$ an den ebenen Schnitt $E_{1}^{n}$ (die zugleich Tangente von $f^{n}$ ist), so steht diese Tangente $B_{1}$ senkrecht auf der Ebene $\left(A_{1} \beta_{1}\right)$; also bildet $B_{1}$ einen rechten Winkel mit $\pi / \beta_{1}$.

Denkt man sich nun eine zweite Gerade $A_{2}$ durch $\pi$; dreht dann um diese als Axe $f^{n}$ unendlich wenig in die Lage $f_{2}^{n}$, so erhält man als Durchschnitt von $f^{n}$ und $f_{2}^{n}$ eine Raumcurve $R_{2}^{n^{2}}=\left(f^{n} f_{2}^{n}\right)$, auf welcher auch die $n$ Schnittpunkte $\eta_{2}=\left(f^{\prime \prime} A_{2}\right)$ liegen. Irgend ein Punkt derselben sei $\beta_{2}$, so ist das Loth $B_{2}$, in $\beta_{2}$ auf der Ebene $\left(A_{2} \beta_{2}\right)$ errichtet, eine Tangente an $f^{n}$ in $\beta_{2}$, die senkrecht steht auf $\pi \beta$.

Betrachten wir insbesondre einen Durchschnittspunkt $\xi=\left(\boldsymbol{R}_{1}^{n^{2}} \boldsymbol{R}_{2}^{n^{2}}\right)$. Denken wir die Ebenen $\left(\xi A_{1}\right)$ und $\left(\xi A_{2}\right)$ und errichten in $\xi$ Lothe auf beiden: $T_{1}$ und $T_{2}$, so sind diese Geraden nach dem Vorigen Tangenten an $f^{n}$ in $\xi$, die beide senkrecht stehen auf $\pi \xi$. Wenn nun nicht $\xi$ in $\operatorname{der}$ Ebene $\left(A_{1} A_{2}\right)$ liegt, so können $T_{1}$ und $T_{2}$ nicht in eine Gerade zusammenfallen, und die Ebene $\left(T_{1} T_{2}\right)$ ist Tangentialebene in $\xi$ an $f^{n} ; \pi \xi$ steht senkrecht darauf, d. h. $\pi \xi$ ist Normale von $f^{n}$ in $\xi$.

Liegt dagegen ein Punkt $\xi$ in $\left(A_{1} A_{2}\right)$, so fallen $T_{1}$ und $T_{2}$ zusammen, bestimmen also keine Ebene, und es kaun $\pi \xi$ im Allgemeinen auch keine Normale sein, weil die Ebene $\left(A_{1} A_{2}\right)$ eine willkürliche Ebene durch $\pi$ ist, also im Allgemeinen keine Normalen durch $\pi$ enthält.

Es fragt sich nun, wieviel Punkte $\xi_{0}$ unter allen Punkten $\xi$ enthalten sind, die zugleich in $\left(A_{1} A_{2}\right)$ liegen. Ein Loth auf $\left(A_{1} A_{2}\right)$ in $\xi_{0}$ errichtet, muss offenbar Tangente an $f^{n}$ in $\xi_{0}$ sein, wie umgekehrt jeder Punkt des ebenen Schnitts $\left[\left(A_{1} A_{2}\right) f^{n}\right]$, durch den eine Tangente auf $f^{n}$ geht, die zugleich normal auf $\left(A_{1} A_{2}\right)$ steht, ein Punkt $\xi_{0}$ ist. Die Normalen auf der Ebene $\left(A_{1} A_{2}\right)$ sind die Geraden, die einen unendlich entfernten Punkt $\gamma$ gemein haben. Der Tangentenkegel (Cylinder) von $\gamma$ hat als Ort der Berührungspunkte eine Raumcurve vom Grade $n(n-1)$, nämlich den Durchschnitt von $f^{n}$ und der ersten Polare von $\gamma$ in Bezug auf $f^{n}$. Diese Curve schneidet die Ebene $\left(A_{1} A_{2}\right)$ in $n(n-1)$ Punkten, und dies sind nach dem Obigen die gesuchten Punkte $\xi_{0}$. Die An- 
zahl $x$ aller Normalen von $\pi$ auf $f^{n}$, deren Fusspunkte wir $\xi_{1}$ nennen, ist die Differenz der Anzahl der Punkte $\xi$ und der der Punkte $\xi_{0}$.

Es giebt $n^{3}$ Punkte $\xi, n(n-1)$ Punkte $\xi_{0}$, also

$$
x=n^{3}-n(n-1)=n\left(n^{2}-n+1\right) \text {. }
$$

Das Resultat, das wir bisher erhalten haben, ist demnach:

Von einem beliebigen Punkte $\pi$ lassen sich auf eine Fläche $f^{n}$ im Allgemeinen $n\left(n^{2}-n+1\right)$ Normale $\pi \xi_{1}$ fällen.

II.

Die gegenseitige Lage der Fusspunkte $\xi_{1}$ lässt sich noch näher feststellen.

Durch die sämmtlichen Punkte $\xi$ geht eine Schaar - Schaar von Flächen $n^{\text {ten }}$ Grades. Diese schneiden die Ebene $\left(A_{1} A_{2}\right)$ in einer Schaar-Schaar von

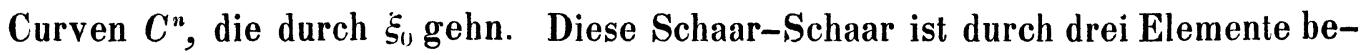
stimmt, z. B. durch die drei ebenen Schnitte von $\left(A_{1} A_{2}\right)$ mit den drei Flächen $f^{n}, f_{1}^{n}, f_{2}^{n}$, die wir bezeichnen durch. $C^{n}, C_{1}^{n}, C_{2}^{n}$. Von dieser Schaar-Schaar geht eine einfache Schaar, ein Büschel, durch $\pi$; um diesen Büschel zu kennen, genügt es zwei beliebige Curven $C$ zu kennen, welche durch $\pi$ gehen. Wir wählen dazu 1) die Curve $C_{3}^{n}$ aus der Schaar $\left(C^{n}, C_{1}^{n}\right)$, d. h. $C_{3}^{n}=\left(C^{n}, C_{1}^{n}\right) \pi$. Da aber ausser $\pi$ die $n$ Punkte $\eta_{1}$ der Axe $A_{1}$ in dieser Curve liegen, so hat $A_{1}$ $(n+1)$ Punkte mit $C_{3}^{n}$ gemein, daher muss $C_{3}^{n}$ aufgelöst sein in die Gerade $A_{1}$ und eine Curve $(n-1)^{\text {ten }}$ Grades durch die Punkte $\xi_{0}$. Wir wählen 2) $C_{4}^{n}=\left(C^{n} C_{2}^{n}\right) \pi$; $C_{4}^{n}$ ist aufgelöst in die Gerade $A_{2}$ und in eine Curve $(n-1)^{\text {ten }}$ Grades. Diese beiden Curven $(n-1)^{\text {ten }}$ Grades sind unter sich identisch, wie aus der Anzahl ihrer gemeinschaftlichen Punkte $\xi_{0}$ hervorgeht. (Sie bilden nämlich beide den Durchschnitt der Ebene $A_{1} A_{2}$ mit der oben betrachteten ersten Polare des Punktes $\gamma$ in Bezug auf $f^{n}$ ). Daraus folgt: Der Flächenbüschel $n^{\text {ten }}$ Grades durch die Punkte $\xi$ und $\pi$ hat zur Fundamentalcurve jene ebene Curve $(n-1)^{\text {ten }}$ Grades und ausserdem eine Raumcurve vom Grade $n^{2}-n+1$, sie heisse $N^{n^{2}-n+1}$. Auf der ersten liegen die Punkte $\xi_{0}$; auf $N$ liegen die Fusspunkte $\xi_{1}$ der Normalen von $\pi$ auf $f^{n}$. Und da alle Sehnen einer Raumcurve $N^{x}$, die durch einen Punkt derselben gezogen sind, einen Kegel vom Grade $(x-1)$ erzeugen, so kann man auch sagen: die Normalen von einem beliebigen Punkte $\pi$ auf $f^{n}$ liegen auf einem Kegel vom Grade $n^{2}-n$. Wir haben also folgenden Satz:

Durch einen beliebigen Punkt $\pi$ lassen sich auf eine Fläche $n^{\text {ten }}$ Grades $f^{n}$ im Allgemeinen $n\left(n^{2}-n+1\right)$ Normalen $\pi \xi_{1}$ fällen. Die Fusspunkte derselben $\xi_{1}$ liegen mit dem Punkte $\pi$ in einer Raumcurve vom Grade $\left(n^{2}-n+1\right)$, nämlich 
$N^{\left(n^{2}-n+1\right)}$; die Normalen $\pi \xi_{1}$ liegen mithin alle auf einem Kegel vom Grade $\left(n^{2}-n\right)$.

Dies lässt sich auch so aussprechen:

Die Normalen einer Fläche $n^{\text {ten }}$ Grades $f^{n}$ bilden ein Strahlensystem von der Ordnung $n\left(n^{2}-n+1\right)$ und von der Klasse $n(n-1)$.

(Dass in jeder Ebene $e n(n-1)$ Normalen von $f^{n}$ liegen, folgt wie oben aus der Betrachtung der ersten Polare des Punktes $\gamma$ in Bezug auf $f^{n}$, wo $\gamma$ der gemeinschaftliche unendlich entfernte Punkt der Geraden ist, die senkrecht auf $e$ stehen. Die Normalen auf $f^{n}$ in den Durchschnittspunkten dieser Polare mit $f^{n}$ und $e$ sind die in $e$ liegenden.)

Im Besondern lassen sich auf eine Fläche zweiten Grades sechs Normalen $\pi \xi_{1}$ von jedem Punkte $\pi$ fällen, und die sieben Punkte $\pi$ und $\xi_{1}$ liegen in einer Raumcurve dritten Grades $N^{3}$; die sechs Normalen $\pi \xi_{1}$ liegen mithin auf einem Kegel zweiten Grades. Sämmtliche Normalen einer Fläche zweiten Grades bilden ein Strahlensystem sechster Ordnung und zweiter Klasse.

Auf eine Fläche dritten Grades lassen sich von einem beliebigen Punkte $\pi 21$ Normalen $\pi \xi_{1}$ fällen; die 22 Punkte $\pi$ und $\xi_{1}$ liegen auf einer Raumcurve siebenten Grades $N^{7}$; die 21 Normalen $\pi \xi_{1}$ liegen mithin auf einem Kegel sechsten Grades. (Das letztere ist hier und bei höheren Graden ohne Interesse, da ein Kegel sechsten Grades erst durch 27 Generatrices bestimmt ist, also durch jene 21 nicht bestimmt wird.) Sämmtliche Normalen einer Fläche dritten Grades bilden ein Strahlensystem einundzwanzigster Ordnung und sechster Klasse u. s. f.

Berlin, im August 1867. 